\title{
AVALIAÇÃO DO PERFIL DOS EGRESSOS DO PROGRAMA DE PÓS GRADUAÇÃO EM ENGENHARIA MINERAL DA UNIVERSIDADE FEDERAL DE OURO PRETO
}

DOI: 10.37702/2175-957X.COBENGE.2021.3397

Carlos Alberto Pereira - pereiraufop@gmail.com

UFOP

Rua João XXIII 240

35400-000 - Ouro Preto - MG

karine fernandes rodrigues - karinefernandes.bio@hotmail.com

Universidade Federal de Ouro Preto

Rua Poluux 255

35420-000 - Mariana - MG

Tatiana Barreto dos Santos - tatiana.santos@ufop.edu.br

Universidade Federal de Ouro Preto

Campus UFOP, Departamento de Engenharia de Minas s/n

35400-000 - Ouro Preto - MG

Emilcy das Graças Guimarães Totti - emilcy.totti@aluno.ufop.edu.br Universidade Federal de Ouro Preto

Rua do Cobre 45

35400-000 - Ouro Preto - MG

FRANCIELLE CÂMARA NOGUEIRA - franciellenoguera@yahoo.com.br UFOP

RUA PADRE ROLIM 1897

35400-000 - OURO PRETO - MG

Resumo: Essa pesquisa apresenta uma análise do perfil dos egressos do Programa de Pós-Graduação em Engenharia Mineral da Universidade Federal de Ouro Preto (PPGEM/UFOP). Para tal, foi realizada uma pesquisa que consistiu no envio de um questionário online para os egressos do PPGEM/UFOP. O questionário foi respondido por 79 egressos do programa. Os resultados indicam que, em sua maioria, os egressos que cursaram o curso de pós-graduação em engenharia 
mineral obtiveram o título de mestre, moram no estado de Minas Gerais, estudaram em instituição de ensino público durante o ensino médio, realizaram graduação na UFOP e foram bolsistas. Com relação a suas atividades atuais, os egressos são, em sua maioria, servidores públicos e atuam como docentes em instituições públicas de ensino. Além disso, os mesmos consideram que a formação recebida no PPGEM foi excelente e que o aprendizado influenciou positivamente em suas práticas profissionais.

Palavras-chave: Engenharia Mineral. Pós-Graduação. Perfil de Egresso. 


\section{AVALIAÇÃO DO PERFIL DOS EGRESSOS DO PROGRAMA DE PÓS GRADUAÇÃO EM ENGENHARIA MINERAL DA UNIVERSIDADE FEDERAL DE OURO PRETO}

\section{INTRODUÇÃO}

Nas últimas décadas, o setor mineral vem buscando melhorias em seus processos operacionais visando maior segurança e sustentabilidade social, ambiental e financeira. Diante desse cenário, os profissionais que fazem parte desse setor identificaram a necessidade de constante atualização e aprimoramento de seus conhecimentos técnicos. Esse fato fez com que os mesmos buscassem em programas de pós-graduação lato sensu, o desenvolvimento de pesquisas voltadas às melhorias dos processos industriais.

O Programa de Pós-Graduação em Engenharia Mineral da Universidade Federal de Ouro Preto (PPGEM-UFOP) teve início no ano de 1998. Desde então, o principal objetivo do programa consiste na formação acadêmica em níveis de mestrado e doutorado, qualificando os egressos nos fundamentos e aplicações da Engenharia Mineral, distribuídos nas áreas de Lavra de Minas e Tratamento de Minérios (CPPGEM, 2020).

De acordo com uma pesquisa realizada por Figueiredo et al. (2020), do início do programa de pós graduação até o ano de 2019, o número de alunos formados em mestrado e doutorado pelo PPGEM-UFOP totalizou um montante de 250 egressos. Segundo os autores, o PPGEM visa preparar os alunos para a atuação no mercado profissional, buscando uma constante integração entre a graduação, pós-graduação, pesquisa e extensão. Dessa forma, além de preparar os mestrandos e doutorandos para a atuação no mercado profissional, o programa também atua na formação didática e acadêmica dos mesmos, desenvolvendo-os para o exercício de atividades em instituições de ensino e/ou pesquisa.

Nesse contexto, o presente estudo avaliou o perfil do egresso do Programa de Pós Graduação em Engenharia Mineral da Universidade Federal de Ouro Preto. Para isso, foi realizado um levantamento de dados a partir de um questionário online, respondido pelos egressos. Os resultados obtidos foram analisados e o perfil dos mesmos foi traçado.

\section{METODOLOGIA}

Com objetivo de traçar o perfil dos egressos, os mesmos foram convidados para participarem da pesquisa, via e-mail, e a responderem um questionário online. A Figura 1 apresenta, de forma esquemática, a metodologia aplicada para obtenção dos dados e avaliação do perfil do egresso do PPGEM/UFOP e o Quadro 1 ilustra o questionário respondido pelos egressos.
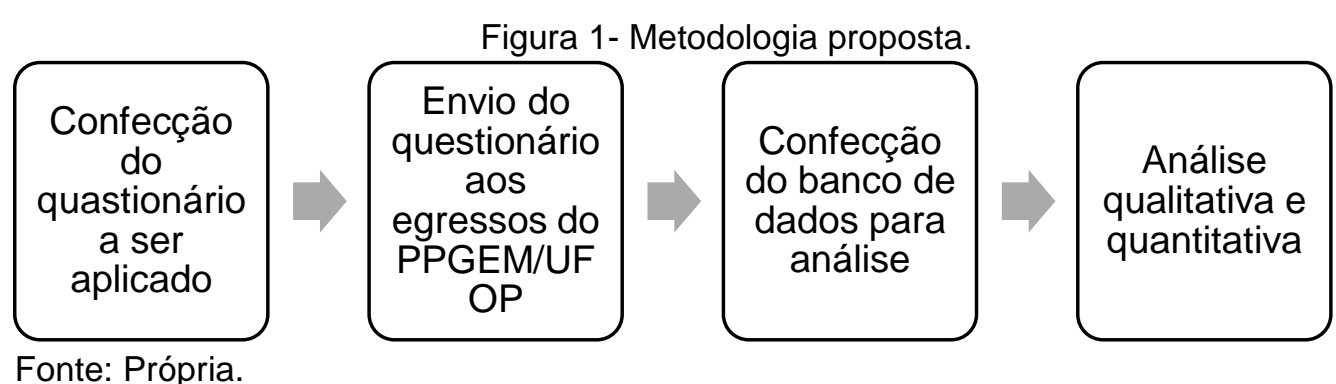

Traçado do perfil do egresso do PPGEM/UF OP

Fonte: Própria. 
Quadro 1- Questionário respondido pelos egressos.

\section{Título obtido}

Mestre

2. Data de titulação dia/mês/ano

3. Residência atual

Brasil Outros Estados

Exterior

\section{Doutor}

\begin{tabular}{|c|c|c|c|c|}
\hline \multicolumn{5}{|c|}{$\begin{array}{l}\text { dia/mês/ano } \\
\text { 3. Residência atual }\end{array}$} \\
\hline Brasil & Minas Gerais & Outros Esta & & Exterior \\
\hline \multicolumn{5}{|c|}{ 4. Onde finalizou o ensino médio? } \\
\hline Em instituição & e ensino pública & Em ins & ão & ensino privada \\
\hline \multicolumn{5}{|c|}{ 5. Onde realizou a graduação? } \\
\hline \multicolumn{2}{|r|}{$\begin{array}{l}\text { Em outra } \\
\text { ensino su }\end{array}$} & $\begin{array}{l}\text { nstituição de } \\
\text { Jerior pública }\end{array}$ & \multicolumn{2}{|c|}{$\begin{array}{l}\text { Em outra instituição de } \\
\text { ensino superior privada }\end{array}$} \\
\hline \multicolumn{5}{|c|}{ 6. Onde realizou o mestrado? } \\
\hline \multicolumn{3}{|c|}{$\begin{array}{l}\text { Em outra instituição de } \\
\text { ensino superior pública }\end{array}$} & \multicolumn{2}{|c|}{$\begin{array}{l}\text { Em outra instituição de } \\
\text { ensino superior privada }\end{array}$} \\
\hline \multicolumn{5}{|c|}{ 7. Onde realizou o doutorado? } \\
\hline UFOP & $\begin{array}{c}\text { Em outra instituição } \\
\text { de ensino superior } \\
\text { pública }\end{array}$ & $\begin{array}{r}\text { Em outra inst } \\
\text { de ensino su } \\
\text { privada }\end{array}$ & & Não se aplica \\
\hline
\end{tabular}

8. Você foi bolsista?

Sim

9. Qual o seu vínculo atual?

Autônomo

Emprego regime CLT

10.Setor de atuação atual

Acadêmico Industrial

Governamental Serviços

Serviço público

Não

\section{Função atual}

Pesquisador

Gestor Consultor Docente em institutos de pesquisa

\begin{tabular}{c|c}
$\begin{array}{c}\text { Em } \\
\text { Doutoramento } \\
\text { (outras } \\
\text { instituições) }\end{array}$ & $\begin{array}{c}\text { Outras } \\
\text { funções }\end{array}$ \\
\hline
\end{tabular}

12. Como você classifica a importância do PPGEM na sua formação para atuação no mercado de trabalho?

Excelente

Satisfatória

Insatisfatória

\section{Ótimo 13. Qual a sua avaliação geral do PPGEM? \\ 14. 0 que você acha que faltou na sua formação para atividade que você exerce hoje?}

Fonte: Própria.

Questão dissertativa

Os dados obtidos foram compilados e submetidos a uma análise quantitativa e qualitativa. Ao final da análise, o perfil do egresso do Programa de Pós Graduação em Engenharia Mineral foi traçado.

\section{RESULTADOS E DISCUSSÃO}

Dos 250 egressos, foram recebidos 79 questionários respondidos, havendo uma aderência de $31,6 \%$ na participação da pesquisa realizada. Em relação ao título obtido, considerando a amostra de egressos respondentes do curso de Pós-Graduação em Engenharia Mineral da Universidade Federal de Ouro Preto, 69 egressos obtiveram título 
de mestre (87,3\%) e 10 egressos obtiveram título de doutor (12,7\%), como mostra a Figura 2. O número baixo de egressos que obtiveram título de doutor nessa amostra pode estar relacionado ao ano de inauguração do doutorado no PPGEM/UFOP, que ocorreu no ano de 2015.

Figura 2: Títulos obtidos pelos egressos do PPGEM/UFOP

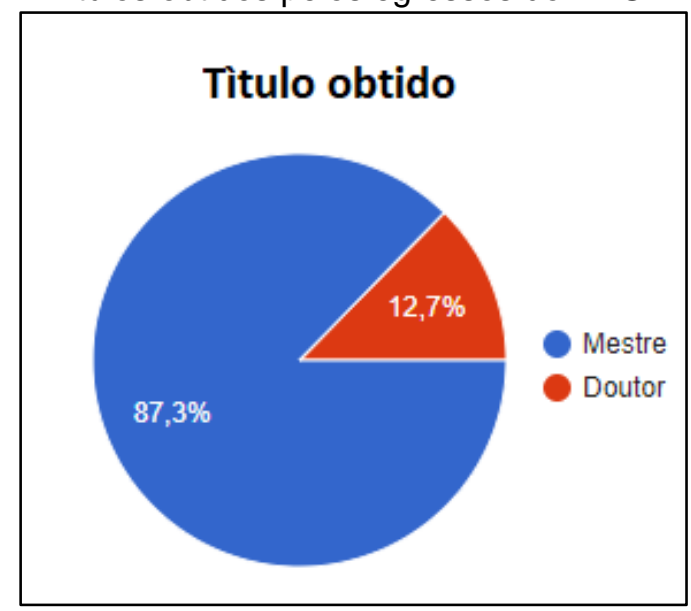

Fonte: Própria.

A Figura 3 mostra os dados referentes à residência atual dos egressos. Dos 79 egressos participantes da pesquisa, 51,9\% residem no Estado de Minas Gerais. Esse fato pode estar associado ao fato de estarmos localizados em uma província mineral. Além disso, 8,9\% moram no exterior. Essa informação, pode sugerir a possibilidade de estar ocorrendo "fuga de cérebros", uma vez que a pesquisa é mais valorizada em outros países.

A Figura 4, mostra os dados obtidos sobre a formação acadêmica dos egressos. Em relação à formação básica, a maioria dos egressos do PPGEM/UFOP, finalizaram o ensino médio em instituição de ensino público $(57,1 \%)$. Com relação à formação superior, em níveis de graduação e pós-graduação, a maioria dos egressos se formaram na UFOP, sendo $66,7 \%$ na graduação, $97,5 \%$ no mestrado e $19,7 \%$ no doutorado, desconsiderando os egressos no qual essa formação não se aplica $(69,7 \%)$. Entre os 79 egressos que responderam o formulário, 51 egressos receberam bolsa de algum orgão de fomento. Esses resultados sugerem que o aluno formado em Engenharia de Minas pela Universidade Federal de Ouro Preto, dá contiuidade à sua formação em sua instituição de origem.

Figura 3 - Residência atual dos egressos do PPGEM/UFOP.

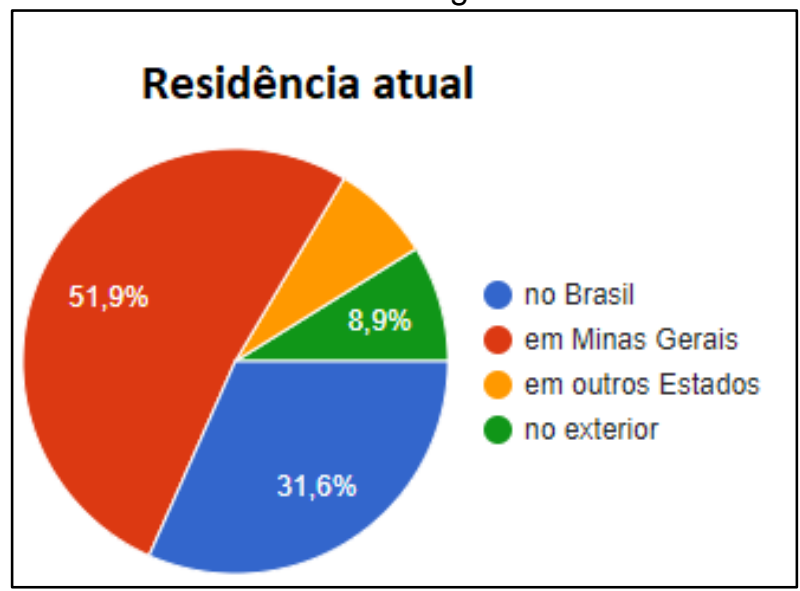

Fonte: Própria. 
Figura 4 - Formação acadêmica dos egressos do PPGEM/UFOP

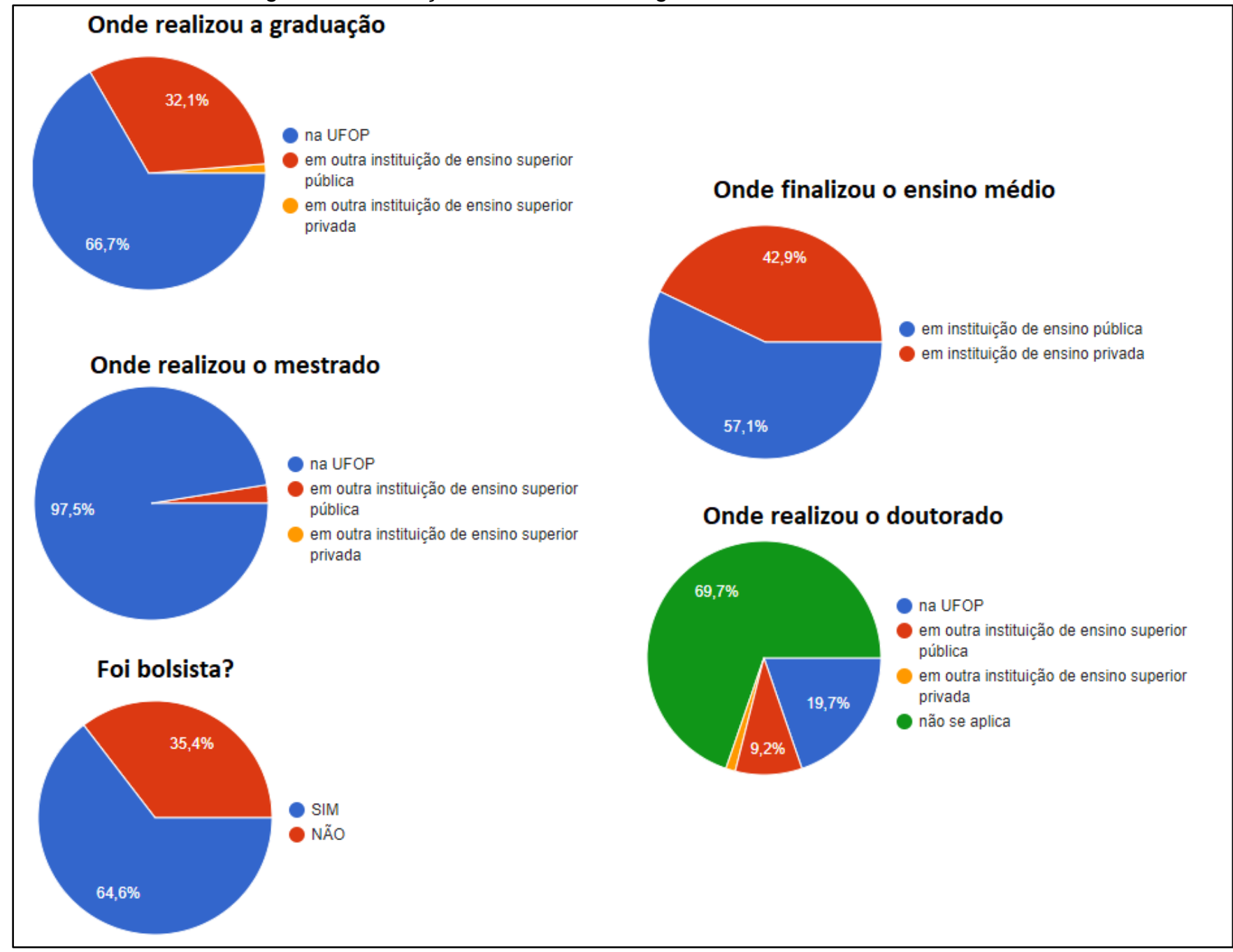

Fonte: Própria.

Com relação à atuação profissional atual dos egressos, a Figura 5 mostra que, o egresso do PPGEM, em sua maioria, atua como servidor público (48,7\%), no setor acadêmico (43\%) e exercendo função de docente (32,9\%). Em relação à função, é interessante observar que, após a função docente, a segunda função com maior atuação dos egressos é a de gestores na indústria (16,5\%). Esses números demonstram que a formação recebida pelos egressos do programa os habilitou a atuar tanto no setor acadêmico quanto no setor industrial.

O estudo realizado por Gunther e Spagnolo (1986), na década de 80, 70\% dos alunos de pós-graduação trabalhavam em instituições de ensino superior (IES). Segundo Velloso (2004), o principal destino profissional de mestres e doutores que atuavam no país na década de 90, ainda era a universidade. No entanto, dados atuais do Conselho Nacional de Desenvolvimento Científico e Tecnológico (CNPq) mostram que apenas 40\% dos mestrandos e doutorandos no país seguem carreira acadêmica. Esses dados mostram que, embora no Brasil os programas de pós graduação tem como principal objetivo o desenvolvimento da pesquisa no meio acadêmico, atualmente a sua atuação tem sido cada 
vez mais importante no desenvolvimento de profissionais que atuam em empresas, capacitando-os e consequentemente elevando o nível técnico do setor mineral como um todo.

Figura 5 - Atuação profissional dos egressos do PPGEM/UFOP.

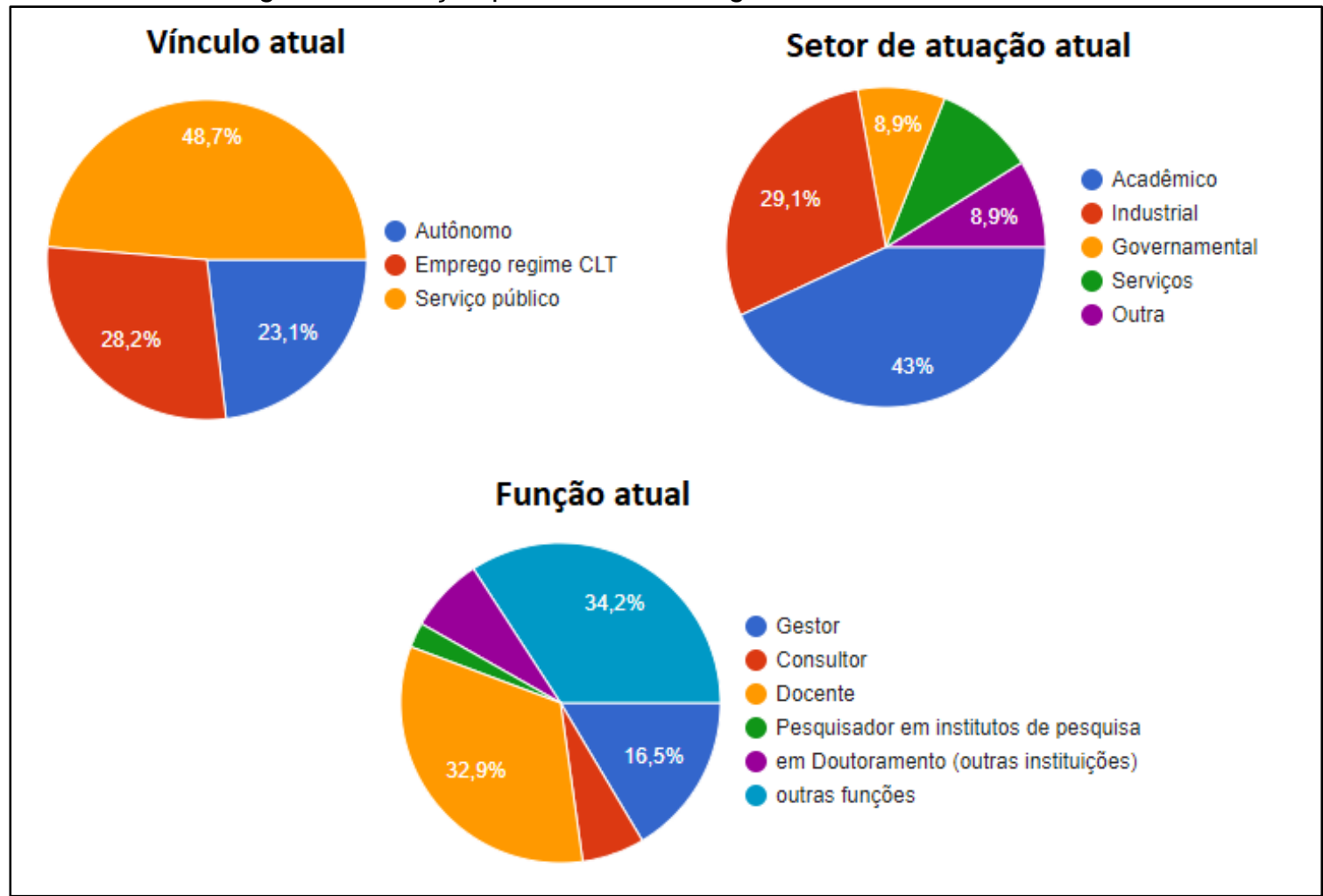

Fonte: Própria.

Em relação à avaliação do PPGEM/UFOP pelos egressos (Figura 6), o PPGEM recebeu evidente avaliação positiva. Dessa forma, considerando a importância do PPGEM na formação de egressos para atuação no mercado de trabalho, 97,4\% dos egressos classificaram o programa como ótimo ou bom. Em relação à uma avaliação geral, 93,6\% dos egressos avaliaram o programa como ótimo ou bom. Nenhum egresso que preencheu o formulário avaliou de forma negativa o PPGEM/UFOP.

Figura 6 - Avaliação do PPGEM/UFOP pelos egressos do programa

Como você classifica a importância do PPGEM na sua formação para atuação no mercado de trabalho?

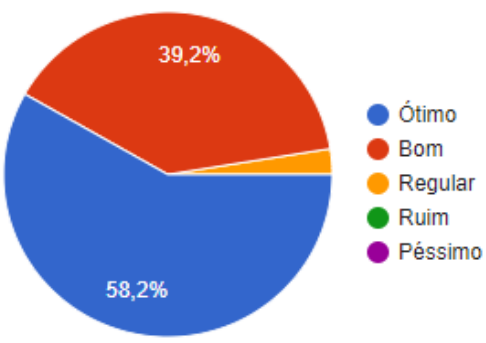

Qual a sua avaliação geral do PPGEM?

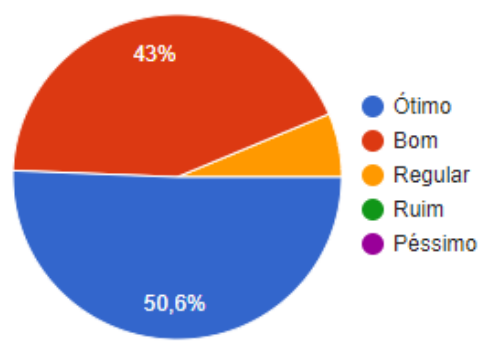

Fonte: Própria. 


\section{CONCLUSÃO}

Os resultados obtidos apontaram que os egressos apresentaram um perfil bem definido. Eles indicaram que, em sua maioria, os egressos que cursaram obtiveram o título de mestre, moram no estado de Minas Gerais, estudaram em instituição de ensino público durante o ensino médio, realizaram graduação na UFOP e foram bolsistas. Com relação a suas atividades atuais, os egressos são em sua maioria servidores públicos e atuam como docentes em instituições públicas de ensino. Além disso, eles consideraram, em sua maioria, que a formação recebida no PPGEM foi excelente e que o aprendizado influenciou positivamente em suas práticas profissionais

\section{Agradecimentos}

Os autores agradecem à UFOP, Fundação Gorceix e CNPq pelo apoio à pesquisa e à produção deste artigo, e aos egressos que cordialmente compartilharam informações de grande importância para a produção deste trabalho.

\section{REFERÊNCIAS}

CPPGEM-UFOP - Colegiado do Programa de Pós-graduação em Engenharia Mineral da UniversidadeFederal de Ouro Preto. Sobre o PPGEM. Disponível em:

https://ppgem.ufop.br/sobre. Acesso em: 15/05/2020.

FIGUEIREDO, T. D.; RODRIGUES, G. H. G.; RODRIGUES K. F.; PEREIRA, A. C. O perfil dos egressos do programa de pós-graduação em engenhariamineral da ufop. In: XLVIII Congresso Brasileiro de Educação em Engenharia E III Simpósio Internacional de Educação em Engenharia da ABENGE, 2020, Bento Gonçalves/RS. Disponível em: http://abenge.org.br/sis artigo doi.php? $\mathrm{e}=\mathrm{COBENGE \& a=20 \& \textrm {c } = 2 8 6 9}$. Acesso em Abril2020.

VELLOSO, J. Mestres e doutores no país: destinos profissionais e políticas de pósgraduação. Cadernos de Pesquisa, São Paulo, v. 34, n. 123, p. 583-611, 2004.

GUNTHER, H.; SPAGNOLO, F. Vinte anos de pós-graduação: o que fazem nossos mestres e doutores? Ciência e Cultura, Campinas, v. 38, n. 10, p. 1643-1662, 1986.

\section{EVALUATION OF THE PROFILE OF GRADUATES OF THE MINERAL ENGINEERING POSTGRADUATE PROGRAM OF THE FEDERAL UNIVERSITY OF OURO PRETO}

Abstract: This research presents an analysis of the graduates profile of The Postgraduate Program in Mineral Engineering of the Federal University of Ouro Preto (PPGEM-UFOP). To this end, a research was carried out which consisted of sending an online questionnaire to the graduates of PPGEM / UFOP. The questionnaire was answered by 79 graduates of the program. The results indicate that, in most cases, the graduates who attended the PPGEM / UFOP obtained the title of master, live in the state of Minas Gerais, studied in a public education institution during high school, graduated from UFOP and were scholarship holders. With regard to their current activities, graduates are mostly civil servants and act as teachers in public educational institutions. In addition, they consider that academic 
education at PPGEM was excellent and that learning had a positive influence on their professional practices.

Keywords: Mineral engineering. Postgraduate program. Graduates profile. 